\title{
Duodenum/Ampulla/Jejunum/Ileum Neuroendocrine Tumor pN1 TNM Finding v7
}

National Cancer Institute

\section{Source}

National Cancer Institute. Duodenum/Ampulla/Jejunum/lleum Neuroendocrine Tumor pN1 TNM Finding v7. NCI Thesaurus. Code C90094.

Duodenum/ampulla/jejunum/ileum neuroendocrine tumor with regional lymph node metastasis. (from AJCC 7th Ed.) 